\title{
CALIBRATION OF A MODULAR STRAW-TUBE-TRACKER FOR THE COSY-TOF EXPERIMENT*
}

\author{
SedigheH JowZAeE \\ for the COSY-TOF Collaboration \\ The Marian Smoluchowski Institute of Physics, Jagiellonian University \\ Reymonta 4, 30-059 Kraków, Poland \\ and \\ Institute für Kernphysik, Jülich Center for Hadron Physics \\ Forschungszentrum Jülich, 52428 Jülich, Germany
}

(Received July 29, 2013)

The Straw-Tube-Tracker (STT) is an essential detector in the COSYTOF experiment which provides accurate position information of charged particle tracks to study strangeness physics. The STT consists of 2704 straw tubes in 13 double-layers, installed in a vacuum tank behind a liquid hydrogen target. The spatial resolution of $150 \mu \mathrm{m}$ and an efficiency of $98 \%$ has been achieved under experimental condition for the individual straws. The STT performance is crucial for geometrical track reconstruction and could be improved further by optimizing the calibration which will increase the reconstruction efficiency and resolution. The new calibration has included different systematic effect corrections e.g. electronics offset and signal width. Hence, $p p$ elastic scattering events measured in fall 2012 at $2.95 \mathrm{GeV} / c$ beam momentum were analyzed for the improved calibration of the STT. The spatial resolution of the COSY-TOF STT could be improved by about $20 \%$ with the new calibration.

DOI:10.5506/APhysPolBSupp.6.1067

PACS numbers: 29.40.-n, 06.20.fb

\section{Introduction}

The $p p \rightarrow p K^{+} \Lambda \rightarrow p K^{+} p \pi^{-}$reaction has been measured with the COSY-TOF spectrometer at $2.95 \mathrm{GeV} / c$ beam momentum in fall 2012. The STT which is used for track reconstruction in the COSY-TOF spectrometer

* Presented at the Symposium on Applied Nuclear Physics and Innovative Technologies, Kraków, Poland, June 3-6, 2013. 
gives a precise direction measurement within the COSY-TOF and is designed to reconstruct the decay vertex. Therefore, it is the most important subsystem for the $p k \Lambda$ analysis and the calibration of the STT is particularly important for the further analysis [1, 2]. To obtain a precise calibration for the STT, some corrections were done on each single straw TDC spectrum. Finally, the correlation between measured drift times and the isochrone radii were deduced from the corrected TDC spectra.

\section{Experimental setup}

The COSY-TOF spectrometer is an external experiment at the Jülich COoler SYnchrotron (COSY). The detector components are inside a $3 \mathrm{~m}$ long vacuum vessel of $2.5 \mathrm{~m}$ diameter. The STT is installed about $30 \mathrm{~cm}$ behind the target and consists of 2704 straw tubes with $10 \mathrm{~mm}$ diameter and $1050 \mathrm{~mm}$ length. The tubes are filled with $\mathrm{Ar} / \mathrm{CO}_{2}$ gas at 1.2 bar overpressure, and are organized in 13 double-layers mounted in three orientations with $60^{\circ}$ angle to each other for 3D track reconstruction [3].

The reconstruction of events with the STT reduces background for better resolution and provides precise reconstruction of the vertices. Because the event analysis is based on the vertices reconstruction of the charged final states, a precise calibration of the STT is needed.

\section{Calibration}

To reach a precise calibration, some corrections were done on each single straw raw TDC spectrum including selection of first hit, signal width cut and electronics offset correction [4].

When a charged track passes the straw tube, gas molecules are ionized. Several ionization clusters are created along this track and can be recorded using fast shaping electronics, but the important timing information is contained in the cluster which is closest to the wire and corresponds to the minimum drift time. Therefore, the first hit was chosen for the time definition in the TDC spectrum. In order to remove the remaining noise hits, the width between the leading and following trailing edge times was calculated and was cut for less than the 5 ns limit of the electronics readout. Next, the spectra were analyzed for electronics offset correction which is largely related to different readout modules. For details of the corrections, see Ref. [4]. The main point of the calibration is the determination of the correlation between the drift time and the isochrone radius which is the radius of closest approach of a particle track to the wire. The $r(t)$ curve was determined with the so-called "self calibrating" method as described in Ref. [5]. An example of the $r(t)$ curve is shown in Fig. 1. This $r(t)$ curve 


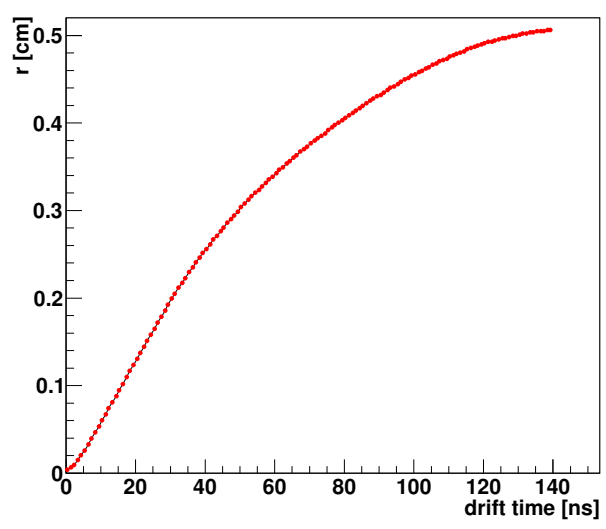

Fig. 1. Isochrone radius versus drift time.

was used in the calibration with the reconstructed track to find the most probable correlation between drift times and track to wire distance. Therefore, track parameters were analyzed with an iterative procedure which is called "autocalibration" [5]. In this method, tracks which are reconstructed as straight lines were analyzed to minimize the track residuals. The residual was defined as the difference of the distance of the best fitted track to straw wire and the isochrone radius predicted from the $r(t)$ curve. If the $r(t)$ curve is correct, the mean residual would be zero at all radii. Figure 2 (left) shows the residual distribution versus the drift time, and the residual distribution in one radius bin is shown in Fig. 2 (right). The mean residual
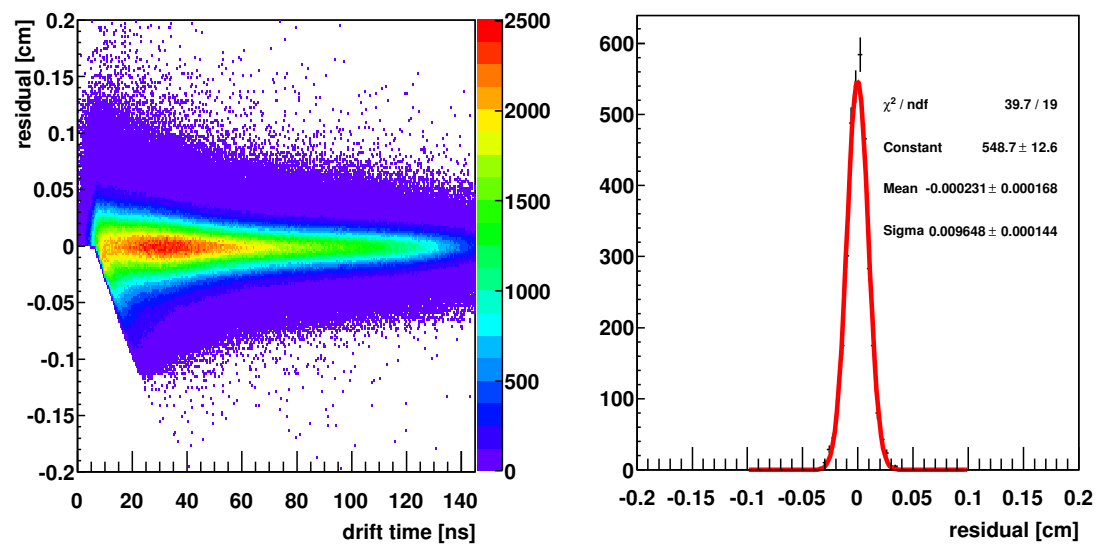

Fig. 2. Left: Distribution of the residual as a function of the measured drift time after five iterations of the autocalibration process. Right: Distribution of the residuals of reconstructed tracks fitted with a Gaussian function (solid/red line). 
distribution was found close to zero as shown in Fig. 3 (left). The spatial resolution of STT was defined as the sigma of the residual distribution of the reconstructed tracks to the isochrone radius. The resolution in all radius bins is shown in Fig. 3 (right). Based on this figure, the resolution is worse close to the wire. This is due to the primary ionization cluster spacing and higher drift velocities close to the wire. Both effects are reduced far from the wire and the resolution improves to about $100 \mu \mathrm{m}$ close to the straw wall.
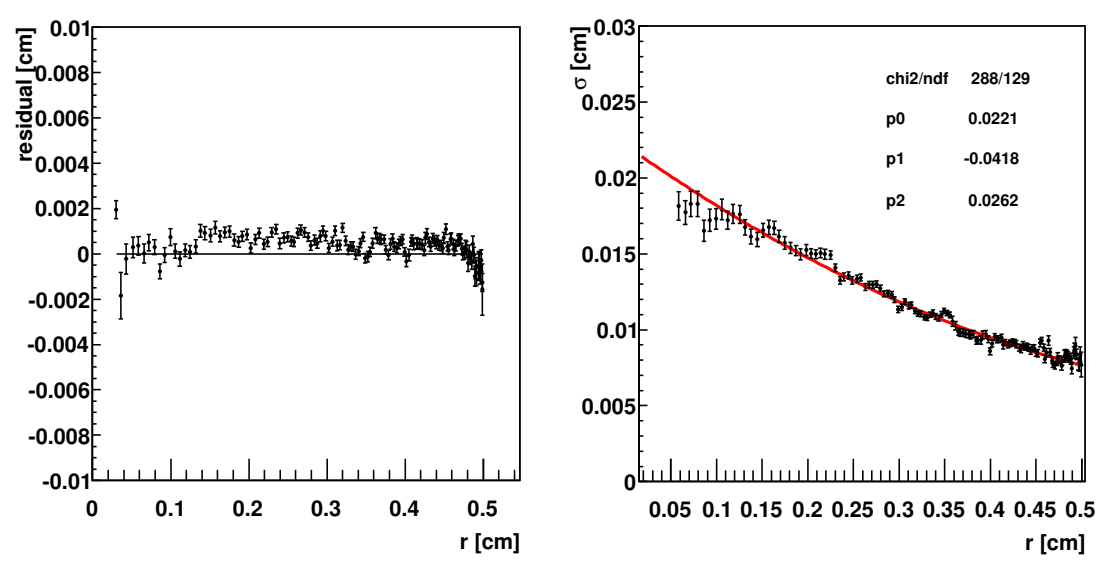

Fig. 3. Left: The mean residuals distribution versus isochrone radius after five iterations. Right: Sigma of the residual distribution to isochrone radius. The resolution distribution is fitted with a second order polynomial function (solid/red line).

\section{Conclusion}

The new calibration shows that applying the signal width cut was effective to clean noisy hits in TDC spectra and the systematic error of different electronics module could be removed significantly by applying the electronics offset correction. A comparison of the mean residual distribution to the former calibration with data at the same beam momentum shows a clear improvement. A spatial resolution of $142 \pm 9 \mu \mathrm{m}$ at a straw radius of $0.25 \mathrm{~cm}$ averaged over all double layers has been obtained with the new calibration compared to the resolution of $174 \pm 18 \mu \mathrm{m}$ [2] with the old calibration. The new calibration are applied for studies of hyperon production with the COSY-TOF STT in the $p p \rightarrow p K^{+} \Lambda$ reaction measured in fall 2012 .

We acknowledge support by the Foundation for Polish Science - MPD program, co-financed by the European Union within the European Regional Development Fund and by the FFE grants of the Research Center Jülich. 


\section{REFERENCES}

[1] R. Dzhygadlo, Ph.D. Dissertation, University of Bonn, 2012.

[2] M. Röder, Ph.D. Dissertation, University of Bochum, 2011.

[3] P. Wintz for the COSY-TOF Collaboration, "A Large Tracking Detector in Vacuum Consisting of Self-supporting Straw Tube", 8th Conference of Intersections of Particle and Nuclear Physics, American Institute of Physics, 2003.

[4] S. Jowzaee for the COSY-TOF Collaboration, IKP Research Center Jülich, Annual Report 2012.

[5] PANDA Collaboration, Eur. Phys. J. A49, 25 (2013) [arXiv: 1205.5441 [physics.ins-det]]. 